\title{
Surface critical behavior of semi-infinite systems with cubic anisotropy at the ordinary transition.
}

\author{
Z. Usatenko ${ }^{1, *}$ and J. Spałek ${ }^{2}$ \\ ${ }^{1}$ Institute for Condensed Matter Physics of the National Academy of Sciences of Ukraine, 79011 Lviv, Ukraine* and \\ ${ }^{2}$ Marian Smoluchowski Institute of Physics, Jagiellonian University, 30-059 Krakow, Poland
}

(Dated: November 21, 2018)

\begin{abstract}
The critical behavior at the ordinary transition in semi-infinite $n$-component anisotropic cubic models is investigated by applying the field theoretic approach in $d=3$ dimensions up to the twoloop approximation. Numerical estimates of the resulting two-loop series expansions for the critical exponents of the ordinary transition are computed by means of Padé resummation techniques. For $n<n_{c}$ the system belongs to the universality class of the isotropic $n$-component model, while for $n>n_{c}$ the cubic fixed point becomes stable, where $n_{c}<3$ is the marginal spin dimensionality of the cubic model. The obtained results indicate that the surface critical behavior of the semi-infinite systems with cubic anisotropy is characterized by a new set of surface critical exponents for $n>n_{c}$.

PACS numbers: PACS number(s): 64.60.Fr, 05.70.Jk, 68.35.Rh, 75.40.Cx
\end{abstract}

\section{INTRODUCTION}

The investigation of the critical behavior of real systems is an important task of the condensed matter theory. The critical behavior in such systems as polymers, easy-axis ferromagnets, superconductors, as well as superfluid ${ }^{4} \mathrm{He}$, Heisenberg ferromagnets, and quark-gluon plasma is described by isotropic $O(n)$ model with $n=0,1,2,3$ and 4 , respectively, and has been analyzed in the framework of different theoretical and numerical approaches.

Investigation of the critical behavior of real cubic crystals is one of the topics of extensive theoretical work during the last three decades. In crystals, due to their crystalline structure, some kind of anisotropy is always present. One of the simplest examples is the cubic anisotropy. A typical model of the critical behavior of such systems is the model with a cubic term $\frac{v_{0}}{4 !} \sum_{i=1}^{n} \phi_{i}^{4}$ added to the usual $O(n)$ symmetric $\frac{u_{0}}{4 !}\left(\sum_{i=1}^{n}\left|\phi_{i}\right|^{2}\right)^{2}$ term ${ }^{1,2,3}$. This $n$-component cubic model is a particular case of an $m n$-component model $^{4}$, with the cubic anisotropy at $m=1$. The model exhibits several types of continuous and first-order phase transitions depending on the number of spin components $n$, space dimensionality $d$, and the sign of the cubic coupling constant $v_{0}$. The cubic models are widely applied to the study of magnetic and structural phase transitions. In the limiting case of $n \rightarrow 0$, it describes the critical behavior of random Ising-like systems ${ }^{5}$. The case $m=0$ and $n=0$ formally describes the critical behavior of long flexible polymer chains in good solvents as model of self-avoiding walks (SAW) on a regular lattice, with short range correlated quenched disorder. As has been shown by Harris ${ }^{6}$ and $\mathrm{Kim}^{7}$, the short range correlated (or random uncorrelated pointlike) disorder is irrelevant for such model. The case $m=1$ and $n \rightarrow \infty$ corresponds to the Ising model with equilibrium magnetic impurities ${ }^{8}$.

Depending on the sign of the cubic coupling constant $v_{0}$, the two types of order are possible: along the diagonals the type $[1,1, \ldots, 1]$ of a hypercube in $n$ dimensions for $v_{0}>0$ or along the easy axes of the type $[1,0, \ldots 0]$ for $v_{0}<0$. In the latter case the system can undergo the first order phase transition, as was confirmed in experiments ${ }^{9}$. In the present work we are concerned with the case $v_{0}>0$.

The presence of a surface leads to the appearance of additional complications. The source of these problems is connected with both the loss of translational invariance and the presence of a boundaries. General reviews on surface critical phenomena are given in Refs. ${ }^{10,11,12}$. The simplest model of critical phenomena in systems with a single planar surface is the semi-infinite model ${ }^{10}$. As it is known ${ }^{10,11,13}$, the phase diagram of such model is richer than that of its bulk correspondant. In general case of pure semi-infinite model with continuous $O(n)$ symmetries, there are a surface- and bulk-disordered phases (SD and BD, respectively), as well as either the surface-ordered, bulk-disordered phase ( $\mathrm{SO}$ and $\mathrm{BD}$, respectively) and a surface-ordered, bulk-ordered phase ( $\mathrm{SO}$ and $\mathrm{BO}$ ). The surface phase can actually occur, if $d>2$ and $n=1$ or $d>3$ and $n>2$. The boundaries between the phases are the lines of surface, ordinary, and extraordinary transitions which meet at a multicritical point $\left(m_{0}^{2}, c_{0}\right)=\left(m_{0 c}^{2}, c_{s p}^{*}\right)$, representing the special transition and called the special point. Each of the above mentioned transitions is characterized by own fixed point. The constant $c_{0}$ is related to the surface enhancement, which measures the enhancement of the interactions at the surface. The coupling $m_{0}$ is defined in Eq.(2.1) below. The nonexistence of this ordered surface phase assume the occurrence of extraordinary transitions. In the case $n=2$ and $d=3$ a surface transitions of the Kosterlitz-Thouless type is present. We do not consider this type of transition and extraordinary ones, because they have a different nature than the special and ordinary transitions.

In general, there are different surface universality classes, defining the critical behavior in the vicinity of the 
system boundaries, at temperatures close to the bulk critical point $\left(\tau=\left(T-T_{c}\right) / T_{c} \rightarrow 0\right)$. Each bulk universality class divides into several distinct surface universality classes. Three surface universality classes, called respectively ordinary $\left(c_{0} \rightarrow \infty\right)$, special $\left(c_{0}=c_{s p}^{*}\right)$ and extraordinary $\left(c_{0} \rightarrow-\infty\right)$, are known ${ }^{11,12,14}$.

In order to investigate the critical behavior of real cubic crystals we must take into account that two types of anisotropy can be present for such systems. The first one is a bulk anisotropy, which can be included into the consideration with a help of above mentioned cubic term. The other one is a surface anisotropy which arise as a consequence of presence the bulk cubic anisotropy (see Appendix A for details). In the present paper we are interested in investigation of the critical behavior only at the ordinary transition, where the surface orders simultaneously with the bulk. In this case, as was founded by Diehl and Eisenriegler ${ }^{15,16}$, the surface anisotropy is irrelevant.

Theory of critical behavior of individual surface universality classes is very well developed for pure isotropic systems $^{12,14,17,18,19,20}$, the systems with quenched surface-enhancement disorder ${ }^{21,22,23}$ and the systems with a random quenched bulk disorder for both the ordinary and the special surface transitions ${ }^{24,25}$. General irrelevance-relevance criteria of the Harris type for the systems with quenched short-range correlated surface-bond disorder were predicted $i^{21}$ and confirmed by Monte-Carlo calculations ${ }^{22,26}$. Moreover, it was established that the surface critical behavior of semi-infinite systems with quenched bulk disorder is characterized by the new set of surface critical exponents in comparison with the case of pure systems ${ }^{24,25}$.

The remainder of this paper is organized as follows. Section II contains the description of model and further useful background. In Section III the renormalization group approach is described. Section IV contains the calculations of the surface renormalization factor $Z_{\partial \varphi}$ and surface critical exponent $\eta_{\partial \varphi}$ by applying the field theoretic approach directly in $d=3$ dimensions, up to the two-loop order. The numerical estimates of the resulting two-loop series expansions for the critical exponents of the ordinary transition are presented in Section V. The calculations are performed by means of the Padé resummation techniques for the cases $n=3,4,8$ and for the case of $n \rightarrow \infty$, which corresponds to the Ising model with equilibrium magnetic impurities. Section VI contains concluding remarks. The Appendix A contains the Landau-Ginzburg-Wilson functional for a semi-infinite systems of spins with cubic anisotropy. The Appendix B contains the standard surface scaling relations for the case $d=3$.

\section{THE MODEL}

The effective Landau-Ginzburg-Wilson Hamiltonian of the $n$-vector model with cubic anisotropy in the semi-infinite space is given by (see Appendix A)

$$
\begin{aligned}
H(\vec{\phi}) & =\int_{0}^{\infty} d z \int d^{d-1} r\left[\frac{1}{2}|\nabla \vec{\phi}|^{2}+\frac{1}{2} m_{0}^{2}|\vec{\phi}|^{2}\right. \\
& \left.+\frac{1}{4 !} v_{0} \sum_{i=1}^{n}\left|\phi_{i}\right|^{4}+\frac{1}{4 !} u_{0}\left(\sum_{i=1}^{n}\left|\phi_{i}\right|^{2}\right)^{2}\right]
\end{aligned}
$$

where $\vec{\phi}(x)=\left\{\phi_{i}(x)\right\}$ is an $n$-vector field with the components $\phi_{i}(x), i=1, \ldots, n$. Here $m_{0}^{2}$ is the "bare mass", representing a linear measure of the temperature difference from the critical point value. The parameters $u_{0}$ and $v_{0}$ are the usual "bare" coupling constants $u_{0}>0$ and $v_{0}>0$. It should be mentioned that the $d$-dimensional spatial integration is extended over a half-space $\mathbb{R}_{+}^{d} \equiv\left\{\mathbf{x}=(\mathbf{r}, z) \in \mathbb{R}^{d}\right.$, with $\mathbf{r} \in \mathbb{R}^{d-1}$ and $\left.z \geq 0\right\}$, bounded by a planar free surface at $z=0$. The fields $\phi_{i}(\mathbf{r}, z)$ satisfy the Dirichlet boundary condition $\phi_{i}(\mathbf{r}, z)=0$ at $z=0$ in the case of ordinary transition, and the Neumann boundary condition $\partial_{n} \phi_{i}(\mathbf{r}, z)=0$ at $z=0$ in the case of special transition ${ }^{14,18}$. The model defined in (2.1) is translationally invariant in directions parallel to the external surface, $z=0$. Thus, we shall use mixed representation, i.e. Fourier representation in $d-1$ dimensions and real-space representation in $z$ direction. Therefore, as was mentioned above ${ }^{15,16}$ and shown in Appendix A, no specific surface term will appear in the case of ordinary transition, when the Dirichlet boundary conditions on the surface are assumed.

The added cubic term breaks the $O(n)$ invariance of the model, leaving a discrete cubic symmetry. The model (2.1) has four fixed points: the trivial Gaussian, the Ising one in which the $n$ components are decoupled, the isotropic $(O(n)$-symmetric), and the cubic fixed points. The Gaussian and Ising fixed points are never stable for any number of components $n$. For isotropic systems, the $O(n)$-symmetric fixed point is stable for $n<n_{c}$, whereas for $n>n_{c}$ it becomes unstable. Here $n_{c}$ is the marginal spin dimensionality of the cubic model, at which the isotropic and cubic fixed points change stability, i.e. for $n>n_{c}$, the cubic fixed point becomes stable. The $O(n)$-symmetric fixed point is tricritical. At $n=n_{c}$, the two fixed points should coincide, and logarithmic corrections to the $O(n)$-symmetric critical exponents are present. The calculation of the critical marginal spin dimensionality $n_{c}$ is the crucial point in studing the critical behavior in three-dimensional cubic crystals. Different results for $n_{c}$ have been published in a series of 
works in which different methods have been used. In the framework of the field-theoretical RG analysis the one-loop and three-loop approximations at $\epsilon=1$ lead to the conclusion that $n_{c}$ should lie between 3 and $4^{33,34}$, and the cubic ferromagnets are described by the Heisenberg model. On the other hand, by using the field theoretic approach directly in $d=3$ dimensions up to the three-loop approximation, it has been found that $n_{c}=2.9^{35,36}$. Similar conclusions were obtained $\mathrm{in}^{37}$, where it was found that $n_{c}=2.3$. The calculations performed by Newman and Riedel ${ }^{38}$ with the help of the scaling-field method, developed by Goldner and Ridel for Wilson's exact momentum-space RG equations, have given for $d=3$ the value $n_{c}=3.4$. Field-theoretical analysis, based on the four-loop series in three dimensions ${ }^{39,40}$ and results of the five-loop $\epsilon=4-d$ expansion ${ }^{40,41,42}$ suggest that $n_{c} \leq 3$. Recently, a very precise six-loop result for the marginal spin dimensionality of the cubic model, $n_{c}=2.89(4)$, was obtained in the framework of the 3D field-theoretic approach ${ }^{43}$. Thus, it was finally established that the critical behavior of the cubic ferromagnets is not described by the isotropic Heisenberg Hamiltonian, but by the cubic model, at the cubic fixed point. However, it was found that the difference between the values of the bulk critical exponents at the cubic and the isotropic fixed points is very small, i.e. it is hard to determine this difference experimentally. Nevertheless, the recently obtained results stimulated us to perform the analysis of the surface critical behavior of semi-infinite $\mathrm{n}$-component anisotropic cubic model, and to determine corresponding surface critical exponents.

\section{RENORMALIZATION}

The fundamental two-point correlation function of the free static theory corresponding to (2.1) is defined by the Dirichlet propagator:

$$
\left\langle\varphi_{i}(r, z) \varphi_{j}\left(0, z^{\prime}\right)\right\rangle_{0}=G_{D}\left(r ; z, z^{\prime}\right) \delta_{i j} .
$$

In the mixed $p z$ representation the Dirichlet propagator is

$$
G_{D}\left(p ; z, z^{\prime}\right)=\frac{1}{2 \kappa_{0}}\left[e^{-\kappa_{0}\left|z-z^{\prime}\right|}-e^{-\kappa_{0}\left(z+z^{\prime}\right)}\right],
$$

where the standard notationis used and, $\kappa_{0}=\sqrt{p^{2}+m_{0}^{2}}$. The propagator vanishes identically when at least one of its $z$ coordinates is zero, because we have assumed the Dirichlet boundary conditions. Consequently, all the correlation functions involving at least one field at the surface vanish. This property holds for both the free and the renormalized theories ${ }^{11}$.

In fact the critical surface singularities at the ordinary transition can be extracted by studying the nontrivial in this case correlation function involving the (inner) normal derivatives of the fields at the boundary, $\partial_{n} \phi(r)^{14,18,46}$. Actually, in order to obtain the characteristic exponent $\eta_{\|}^{\text {ord }}$ of surface correlations, it is sufficient to consider a correlation function with two normal derivatives of boundary fields, i.e.

$$
\mathcal{G}_{2}(p)=\left\langle\left.\left.\frac{\partial}{\partial z} \varphi(p, z)\right|_{z=0} \frac{\partial}{\partial z^{\prime}} \varphi\left(-p, z^{\prime}\right)\right|_{z^{\prime}=0}\right\rangle,
$$

where the fields $\varphi(p, z)$ are the Fourier transforms of the fields $\varphi(r, z)$ in the $(d-1)$ dimensional parallel to surface space. $\mathcal{G}_{2}(p)$ is a parallel Fourier transform of the corresponding two-point function $\mathcal{G}_{2}(r)$ in direct space. At the critical point $\mathcal{G}_{2}(p)$ behaves as $p^{-1+\eta_{\|}^{\text {ord }}}$. It reproduces the leading critical behavior of a two-point function $G_{2}(p)=\left\langle\varphi(p, z) \varphi\left(-p, z^{\prime}\right)\right\rangle$ in the vicinity of the boundary plane. The surface critical exponent $\eta_{\|}^{\text {ord }}$ is provided by the scaling dimension of the boundary operator $\partial_{n} \varphi(r)$.

The surface correlation function exponent $\eta_{\|}^{\text {ord }}$ in the semi-infinite systems with cubic anisotropy differs from its corresponding value for the isotropic semi-infinite system. The remaining surface critical exponents of the ordinary transition can be determined through the surface scaling laws ${ }^{11}$. (see Appendix B).

In the present formulation of the problem, the renormalization process for the cubic anisotropic system is essentially the same as in the isotropic case ${ }^{11,20}$. Explicitly, the renormalized bulk field and its normal derivative at the surface should be reparametrized by different uv-finite renormalization factors $Z_{\varphi}(u, v)$ and $Z_{\partial \varphi}(u, v)$

$$
\varphi_{R}(x)=Z_{\varphi}^{-\frac{1}{2}} \varphi(x) \text { and }\left(\partial_{n} \varphi(r)\right)_{R}=Z_{\partial \varphi}^{-\frac{1}{2}} \partial_{n} \varphi(r),
$$

and renormalized correlation functions involving $N$ bulk fields and $M$ normal derivatives are

$$
\mathcal{G}_{R}^{(N, M)}(p ; m, u, v)=Z_{\varphi}^{-\frac{N}{2}} Z_{\partial \varphi}^{-\frac{M}{2}} \mathcal{G}^{(N, M)}\left(p ; m_{0}, u_{0}, v_{0}\right)
$$


for $(N, M) \neq(0,2)$. In order to remove the ultraviolet (uv) singularities of the correlation function $\mathcal{G}^{(0,2)}$ with two surface operators $(N, M)=(0,2)$ in the vicinity of the surface, an additional, additive renormalization (zeromomentum subtraction) is required, so that

$$
\mathcal{G}_{R}^{(0,2)}(p)=Z_{\partial \varphi}^{-1}\left[\mathcal{G}^{(0,2)}(p)-\mathcal{G}^{(0,2)}(p=0)\right]
$$

The typical bulk uv singularities, which are present in the correlation function $\mathcal{G}^{(0,2)}$, are subtracted via the standard mass renormalization of the massive infinite-volume theory. It also relates to coupling constants, for which standard vertex renormalization of coupling constants takes place.

The surface renormalization factor $Z_{\partial \varphi}(u, v)$ can be conveniently obtained from the consideration of the boundary two-point function $\mathcal{G}^{(0,2)}$

$$
Z_{\partial \varphi}=-\lim _{p \rightarrow 0} \frac{m}{p} \frac{\partial}{\partial p} \mathcal{G}^{(0,2)}(p) .
$$

A standard RG argument involving an inhomogeneous Callan-Symanzik equation yields the anomalous dimension of the operator $\partial_{n} \varphi(r)$

$$
\begin{aligned}
\eta_{\partial \varphi} & =\left.m \frac{\partial}{\partial m} \ln Z_{\partial \varphi}\right|_{F P} \\
& =\beta_{u}(u, v) \frac{\partial \ln Z_{\partial \varphi}(u, v)}{\partial u}+\left.\beta_{v}(u, v) \frac{\partial \ln Z_{\partial \varphi}(u, v)}{\partial v}\right|_{F P}
\end{aligned}
$$

"FP" indicates here that the above value should be calculated at the infrared-stable cubic fixed point of the underlying bulk theory, $(u, v)=\left(u^{*}, v^{*}\right)$. The surface critical exponent $\eta_{\|}^{\text {ord }}$ at the ordinary transition is then given by

$$
\eta_{\|}^{\text {ord }}=2+\eta_{\partial \varphi}
$$

\section{PERTURBATION THEORY UP TO TWO-LOOP APPROXIMATION}

After performing the mass and additive renormalization of the correlation function $\mathcal{G}^{(0,2)}(p)$ and carrying out the integration of Feynman integrals by analogy with Ref. ${ }^{20,24}$, we obtain for renormalization factor

$$
Z_{\partial \varphi}\left(\bar{u}_{0}, \bar{v}_{0}\right)=1+\frac{\bar{t}_{1}^{(0)}}{4}+\bar{t}_{2}^{(0)} C
$$

where the constant $C$ follows from the two-loop (melon-like diagrams) contribution to the correlation function and has the value,

$$
C \simeq \frac{107}{162}-\frac{7}{3} \ln \frac{4}{3}-0.094299 \simeq-0.105063
$$

The coefficients $\bar{t}_{1}^{(0)}$ and $\bar{t}_{2}^{(0)}$ are the weighting factors belonging to one- and two-loop (melon-like) diagrams in the Feynman diagrammatic expansion of the correlation function $\mathcal{G}^{(0,2)}(p)$, and equal

$$
\begin{aligned}
-\frac{\bar{t}_{1}^{(0)}}{2}, \quad \text { with } \quad \bar{t}_{1}^{(0)} & =\frac{n+2}{3} \bar{u}_{0}+\bar{v}_{0}, \\
\frac{\bar{t}_{2}^{(0)}}{6}, & \text { with } \quad \bar{t}_{2}^{(0)}=\frac{n+2}{3} \bar{u}_{0}^{2}+\bar{v}_{0}^{2}+2 \bar{v}_{0} \bar{u}_{0} .
\end{aligned}
$$

The factors $\bar{t}_{1}^{(0)}$ and $\bar{t}_{2}^{(0)}$ follow from the standard symmetry properties of Hamiltonian (2.1). Here the renormalization factor $Z_{\partial \varphi}$ is expressed as a second-order series expansion in powers of bare dimensionless parameters $\bar{u}_{0}=u_{0} /(8 \pi m)$ and $\bar{v}_{0}=v_{0} /(8 \pi m)$. As it is usual in superrenormalizable theories, the renormalization factor expressed in terms of unrenormalized coupling constants, is finite. 
As a next step, the vertex renormalizations should be carried out. To the present accuracy, they are

$$
\begin{aligned}
& \bar{u}_{0}=\bar{u}\left(1+\frac{n+8}{6} \bar{u}+\bar{v}\right), \\
& \bar{v}_{0}=\bar{v}\left(1+\frac{3}{2} \bar{v}+2 \bar{u}\right) .
\end{aligned}
$$

As known, the vertex renormalization at $d=3$ is a finite reparametrization. All relevant singularities have been removed already after the mass renormalization and taking into account the special bubble-graph combinations emerging in the theory with Dirichlet propagators. Thus we obtain a modified series expansion up to two-loop approximation

$$
\begin{aligned}
Z_{\partial \varphi}(\bar{u}, \bar{v}) & =1+\frac{n+2}{12} \bar{u}+\frac{\bar{v}}{4}+\frac{n+2}{3}\left(C+\frac{n+8}{24}\right) \bar{u}^{2} \\
& +\left(C+\frac{3}{8}\right) \bar{v}^{2}+2\left(C+\frac{n+8}{24}\right) \bar{u} \bar{v} .
\end{aligned}
$$

Combining the renormalization factor $Z_{\partial \varphi}(\bar{u}, \bar{v})$ together with the one-loop pieces of the beta functions : $\beta_{\bar{u}}(\bar{u}, \bar{v})=$ $-\bar{u}\left(1-\frac{n+8}{6} \bar{u}-\bar{v}\right)$ and $\beta_{\bar{v}}(\bar{u}, \bar{v})=-\bar{v}\left(1-\frac{3}{2} \bar{v}-2 \bar{u}\right)$ and inserting them into Eq.(3.7), we obtain the desired series expansion for $\eta_{\partial \varphi}$,

$$
\begin{aligned}
& \eta_{\|}(u, v)=2-\frac{n+2}{2(n+8)} u-\frac{v}{6} \\
& -24 \frac{(n+2)}{(n+8)^{2}} \mathcal{C}(n) u^{2}-\frac{8}{9} \mathcal{C}(1) v^{2}-\frac{16}{n+8} \mathcal{C}(n) u v,
\end{aligned}
$$

where $\mathcal{C}(n)$ is a function of the order-parameter components number $n$, and is defined as

$$
\mathcal{C}(n)=C+\frac{n+14}{96}
$$

whereas the renormalized coupling constants $u$ and $v$, normalzed in a standard fashion are $u=\frac{n+8}{6} \bar{u}$ and $v=\frac{3}{2} \bar{v}$.

Eq.(4.8) supplies our result for the critical exponent of surface correlation function for the model with the effective Hamiltonian of the Landau-Ginzburg-Wilson type with cubic anisotropy in the semi-infinite space (2.1) with general number $n$ of order parameter components.

The knowledge of $\eta_{\|}$gives possibility to calculate the other surface critical exponents through the scaling relations. For convenience, from now on we omit the superscript ord for the surface critical exponents.

The critical exponents should be calculated for different $n(n=3,4,8$, and $n \rightarrow \infty)$ at the standard infrared-stable cubic fixed (FP) points of the underlying bulk theory, as is usually accepted in the massive field theory. As was mentioned above, in the cases $n<n_{c}$ the cubic ferromagnets are described by the Heisenberg isotropic Hamiltonian at the $O(n)$-symmetric fixed point.

In the case of the replica limit $n \rightarrow 0$ we obtain from (4.8) the series expansion of $\eta_{\|}^{r}$ for semi-infinite random Ising-like systems. This case was investigated in detail of one of us previously ${ }^{24}$.

\section{NUMERICAL RESULTS}

In order to obtain the full set of surface critical exponents for the ordinary transition in systems with cubic anisotropy, we substitute the expansion (4.8) for $\eta_{\|}$into the standart scaling-law expressions for the surface exponents (see Appendix 1).

For each of the above mentioned surface critical exponents of the ordinary transition we obtain for $d=3$ a double series expansion in powers of $u$ and $v$, truncated at the second order. As it is known ${ }^{58,59,60,61}$, power series expansions of this kind are generally divergent due to a nearly factorial growth of expansion coefficients at large orders of perturbation theory. In order to perform the analysis of these perturbative series expansions and obtain accurate estimates of the surface critical exponents, a powerful resummation procedure must be used. One of the simplest ways is to perform the double Padé-analysis ${ }^{44}$. This should work well when the series behaves in lowest orders "in a convergent fashion".

The results of our calculations of the surface critical exponents of the ordinary transition for various values of $n=3,4,8, \infty$ at the corresponding cubic fixed points are presented in Tables 1-5. Unfortunately, the second-order 
$(\mathrm{p}=2)$ analysis of perturbative series ${ }^{63}$ gives the cubic fixed point with coordinates $u_{0}=1.5347$ and $v_{0}=-0.0674$ at $n=3$ for the $3 \mathrm{D}$ model. The analysis of the eigenvalues of the stability matrix shows that in the frames of the two-loop approximation the cubic fixed point at $n=3$ is unstable and the $O(n)$-symetric fixed point is stable. But, the estimates of the marginal spin dimensionality of the cubic model $n_{c}$ in the frames of three-loop ${ }^{35,36}$, four-loop ${ }^{39,40}$, five-loop $\epsilon=4-d$ expansion ${ }^{40,41,42}$ and six-loop study ${ }^{43}$ show that the cubic ferromagnets are not described by the Heisenberg isotropic model, but by the cubic model at the stable cubic fixed point. Higher precision six-loop fieldtheoretical analysis ${ }^{43}$ give the value of the marginal spin dimensionality of the cubic model equal to $n_{c}=2.89(4)$. In accordance with this we use the cubic fixed point of the higher $p=3$ order of perturbative series for obtaining the set of surface critical exponents at $n=3$. For estimation of the reliability of the obtained results we performed calculations at the cubic fixed point of the $p=6$ order in Table 2. We obtained that difference in these two cases are approximatively: $0.5 \%$ for $\eta_{\|}, 0.4 \%$ for $\eta_{\perp}, 0.8 \%$ for $\Delta_{1}, 0.1 \%$ for $\beta_{1}, 6 \%$ for $\gamma_{11}, 0.2 \%$ for $\gamma_{1}, 0.2 \%$ for $\delta_{1}$, and $0.8 \%$ for $\delta_{11}$. The obtained results indicate that the difference in the ways of the $\beta$ functions resummation have no essential influence on the values of the surface critical exponents and that the results obtained in the frames of the two-loop approximation are stable and reliable. The surface critical exponents of the ordinary transition for $n=4,8$ and $n \rightarrow \infty$ were calculated at the standard infrared-stable cubic fixed (FP) points of the underlying bulk theory, as it is usually accepted in the massive field theory.

The quantities $O_{1} / O_{2}$ and $O_{1 i} / O_{2 i}$ represent the ratios of magnitudes of first-order and second-order perturbative corrections appearing in direct and inverse series expansions. The larger (absolute) value of these ratios indicate the better apparent convergence of truncated series.

The values $[p / q]$ (where $p, q=0,1$ ) are simply Padé approximants which represent the partial sums of the direct and inverse series expansions up to the first and the second order. The nearly diagonal two-variable rational approximants of the types [11/1] and [1/11] give at $u=0$ or $v=0$ the usual [1/1] Padé approximant ${ }^{44}$. As it is easy to see from Tables 1-5, the values of [11/1] and [1/11] Padé aproximants do not differ significantly between themselves. We consider these values as the best we could achieve from the available knowledge about the series expansions in the two-loop approximation scheme. Thus, our final results are presented in the last columns of the Tables 1-5. Their deviations from the other second-order estimates might serve as a rough measure of the achieved numerical accuracy. As it is easy to see, the obtained results indicate about good stability of the results calculated in the frames of the two-loop approximation scheme.

The results for surface critical exponents of semi-infinite model with cubic anisotropy, calculated at the cubic fixed point are different from the results for surface critical exponents of standart semi-infinite $n$-component model $\left(\operatorname{see}^{14,17,20,57}\right)$.

If $n<n_{c}$, the cubic fixed point is unstable and the cubic term in the Hamiltonian (2.1) becomes irrelevant. In this case the isotropic fixed point is stable and the system is described by the simple $O(n)$-symmetric model in 3D. The corresponding surface critical exponents can be calculated from the series, presented $i^{20}$.

As was indicated previously, in the limit $n \rightarrow 0$, the cubic model (2.1) with $u_{0}<0$ and $v_{0}>0$ describes the semi-infinite Ising-like systems with random bulk disorder. The investigation of the ordinary transition for such kind of systems was presented $\mathrm{in}^{24}$.

\section{CONCLUDING REMARKS}

We have studied ordinary transition for a semi-infinite systems with cubic anisotropy by applying the field theoretic approach directly in $d=3$ dimensions, up to the two-loop approximation. We have performed a double Padé analysis of the resulting perturbation series for the surface critical exponents of the ordinary transition for various $n=3,4,8, \infty$, in order to find the best numerical estimates. We find that at $n>n_{c}$ the surface critical exponents of the ordinary transition in semi-infinite systems with cubic anisotropy belong to the cubic universality class.

In order to obtain more precise numerical estimates for the case of $3 \mathrm{D}$ dimensional cubic crystal with $n=3$, a further theoretical investigation of the asymptotic surface critical behavior of semi-infinite cubic systems would be highly desirable within the framework of higher-order RG approximations.

We suggest that the obtained results could stimulate further experimental and numerical investigations of the surface critical behavior of random systems and systems with cubic anisotropy.

\section{Acknowledgments}

One of us (Z.U.) was supported in part by the Queen Jadwiga Fellowship of the Jagiellonian University, Krakow, Poland. The second author (J.S.)acknowledges the support of the State Committee for Scientific Research (KBN), Grant N 2P03B05023, as well as the Fellowship of the Foundation for Science (FNP). . 


\section{Appendix A. The Landau-Ginzburg-Wilson functional for the semi-infinite systems with cubic anisotropy}

We outline the main steps of deriving the Landau-Ginzburg-Wilson functional for the case of Heisenberg ferromagnet and discuss briefly the surface term leading to the surface boundary conditions. We perform the calculation for a simple cubic structure and start from the mean-field approximation for the Heisenberg exchange interaction, as the cubic-anisotropy term can be added afterwards. Additionally, we limit ourselves to one-component theory, as the generalization to the $n$-component version is not important to the essence of the principal argument.

Suppose we have the system of localized spins of magnitude $S$ and described by the Heisenberg Hamiltonian, which in the applied field $h$ takes the form

$$
H=-\frac{1}{2} \sum_{i \neq j} J_{i j} \mathbf{S}_{i} \bullet \mathbf{S}_{j}-h \sum_{i} S_{i}^{z}
$$

In the mean-field approximation

$$
\mathbf{S}_{i} \bullet \mathbf{S}_{j}=<\mathbf{S}_{i}>\bullet \mathbf{S}_{j}+<\mathbf{S}_{j}>\bullet \mathbf{S}_{i}-<\mathbf{S}_{i}>\bullet<\mathbf{S}_{j}>
$$

and for the spin quantization axis taken as the z-axis. We can write the free energy in the form

$$
F=-\tilde{N} k_{B} T \ln \frac{\sinh \left(\beta h_{i}(S+1 / 2)\right)}{\sinh \left(\beta h_{i} / 2\right)}+\frac{1}{2} \sum_{i \neq j} J_{i j}<S_{i}^{z}><S_{j}^{z}>,
$$

where $h_{i}=\sum_{j} J_{i j}\left\langle S_{j}^{z}\right\rangle+h$ is the effective field acting on $S_{i}^{z}, \beta=\left(k_{B} T\right)^{-1}$ is the inverse temperature in energy units, and $\bar{N}$ is the total number of spins.

The constant term can be rewritten in the first nontrivial order the continuum-medium approximation as

$$
\frac{1}{2} \sum_{j} J_{i j}<S_{i}^{z}><S_{j}^{z}>\simeq \frac{1}{2} J_{0}<S^{z}(\mathbf{x})>\left.\right|_{\mathbf{x}=\mathbf{R}_{i}}+\frac{1}{2} a_{0}^{2}<S^{z}(\mathbf{x})>\nabla^{2}<S^{z}(\mathbf{x})>\left.\right|_{\mathbf{x}=\mathbf{R}_{i}}
$$

where $J_{0}=\sum_{j} J_{i j}, a_{0}$ is the lattice constant, and $i=\mathbf{R}_{i}$ denotes here the lattice site position.

The expansion of the ratio of hyperbolic function in $y=\beta h_{i}$ can be represented as

$$
\begin{aligned}
\frac{\sinh (y(S+1 / 2))}{\sinh (y / 2)} & \simeq(2 S+1)\left[1+\frac{1}{6} S(S+1) y^{2}\right. \\
& \left.+\frac{1}{360} S\left(3 S^{3}+6 S^{2}+2 S-1\right) y^{4}\right]+O\left(y^{6}\right)
\end{aligned}
$$

Hence, the free energy (per site) to the same approximation after taking the continuum-medium limit reads

$$
\begin{aligned}
\frac{F}{\tilde{N}} & =\frac{F_{0}}{\tilde{N}}+\int d^{d} x\left(\frac{3}{2 S(S+1)} k_{B}\left(T-T_{c}\right) \phi(\mathbf{x})^{2}\right. \\
& +\frac{1}{2} J_{0} a_{0}^{2}|\nabla \phi(\mathbf{x})|^{2}-\frac{1}{3} S(S+1) \beta h J_{0} \phi(\mathbf{x}) \\
& \left.+\frac{\beta^{3}}{72}\left(S^{2}(S+1)^{2}-\frac{S}{5}\left(3 S^{3}+6 S^{2}+2 S-1\right)\right)\left(J_{0} \phi(\mathbf{x})^{4}\right)\right) \\
& +o\left(|\phi|^{6}\right)+o\left(h^{2}\right)+o\left(|\nabla \phi|^{4}\right),
\end{aligned}
$$

with $\frac{F_{0}}{N}=-k_{B} T \ln (2 S+1)$. The continuous field $\phi(\mathbf{x})$ expresses the limiting value of $\left\langle S_{i}^{z}>\right.$ per volume $a_{0}^{d}$.

Defining the mean-field critical temperature $T_{c}=\frac{1}{3 k_{B}} J_{0} S(S+1)$ and assuming that we can put $T \simeq T_{c}$ in the last two terms we obtain the desired free energy functional in the form

$$
\begin{aligned}
\frac{F}{\tilde{N}} & =\frac{F_{0}}{\tilde{N}}+\int d^{d} x\left(\frac{A_{0}}{2}|\nabla \phi(\mathbf{x})|^{2}+\frac{A_{1}}{2}\left(T-T_{c}\right)|\phi(\mathbf{x})|^{2}\right. \\
& \left.+\frac{A_{2}}{4 !}|\phi(\mathbf{x})|^{4}-h \phi(\mathbf{x})\right),
\end{aligned}
$$

where the constants are related in an obvious fashion to the coefficients $m_{0}^{2}$ and $u_{0}$, when we divide $F$ by the exchange stiffness constant $A_{0}$, which contains non-divergent constants at the critical point. 
Now, the boundary conditions appear when we derive the Landau-Ginzburg equation in the form of Euler equation for $\phi(\mathbf{x})$. However, in such a situation specific surface terms appear. The are two types of terms. First, is the gradient

term $A_{0}^{\prime}|\nabla \phi(\mathbf{r}, z=0)|^{2}$, since in $J_{0}=\sum_{j} J_{i j}$ the spins above the surface are missing. Second, the geometrical surface term of the form $\frac{1}{2} c_{0} \int d^{d-1} r \phi^{2}(\mathbf{r}, z=0)$ may appear where the $c_{0}$ is the surface enhancement constant. This is becouse on the surface the role of the bulk cubic anisotropy is taken over by the surface anisotropy, which in the first nontrivial order has the form $\frac{1}{2} \sum_{i=1}^{n} c_{0}^{i} \phi_{i}^{2}(\mathbf{r}, z=0)$. In effect, the condition on the surface coming from the Euler variational scheme takes the following form

$$
\left.\sum_{i=1}^{n} \int d^{d-1} r\left\{A_{0}^{\prime} \vec{n} \bullet \nabla \phi_{i}(\mathbf{r}, z)+c_{0}^{i} \phi_{i}(\mathbf{r}, z)\right\} \delta \phi_{i}(\mathbf{r}, z)\right|_{z=0}=0
$$

where $\delta \phi_{i}(\mathbf{r}, z=0)$ is the variation of $\phi_{i}(\mathbf{r}, z)$ on the surface and $\vec{n}$ is the vector perpendicular to the surface. Therefore, we can choose the boundary conditions in either way, namely

$$
\begin{array}{ll} 
& \left.\delta \phi_{i}(\mathbf{r}, z)\right|_{z=0}=0, \quad \text { i.e. }\left.\quad \phi_{i}(\mathbf{r}, z)\right|_{z=0}=\text { const }=S_{0}, \\
\text { 2) } & \left.\left(\partial_{n} \phi_{i}(\mathbf{r}, z)+\frac{c_{0}^{i}}{A_{0}^{\prime}} \phi_{i}(\mathbf{r}, z)\right)\right|_{z=0}=0 .
\end{array}
$$

In this paper we have selected the b.c. 1) with $S_{0}=0$, which correspond to the Dirichlet boundary condition. In such a situation, no specific surface term appears in the starting functional (2.1). However, it must be said that it is the concrete experimental situation that determines the type of boundary conditions to be taken into the theoretical analysis.

\section{Appendix B. Scaling relations between the surface critical exponents}

The individual RG series expansions for other critical exponents can be derived through standard surface scaling relations ${ }^{11}$ with $d=3$ :

$$
\begin{aligned}
& \eta_{\perp}=\frac{\eta+\eta_{\|}}{2}, \\
& \beta_{1}=\frac{\nu}{2}\left(d-2+\eta_{\|}\right), \\
& \gamma_{11}=\nu\left(1-\eta_{\|}\right), \\
& \gamma_{1}=\nu\left(2-\eta_{\perp}\right), \\
& \Delta_{1}=\frac{\nu}{2}\left(d-\eta_{\|}\right), \\
& \delta_{1}=\frac{\Delta}{\beta_{1}}=\frac{d+2-\eta}{d-2+\eta_{\|}}, \\
& \delta_{11}=\frac{\Delta_{1}}{\beta_{1}}=\frac{d-\eta_{\|}}{d-2+\eta_{\|}} .
\end{aligned}
$$

Each of these critical exponents characterizes certain properties of the cubic anisotropic system near the surface. The values $\nu, \eta$, and $\Delta=\nu(d+2-\eta) / 2$ are the standard bulk exponents. 
TABLE I: Surface critical exponents of the ordinary transition for $d=3$ up to two-loop order at the cubic fixed point (of order $p=3): u^{*}=1.348, v^{*}=0.074$, at $n=3$.

\begin{tabular}{rrrrrrrrrrr}
\hline $\exp$ & $\frac{O_{1}}{O_{2}}$ & $\frac{O_{1 i}}{O_{2 i}}$ & {$[0 / 0]$} & {$[1 / 0]$} & {$[0 / 1]$} & {$[2 / 0]$} & {$[0 / 2]$} & {$[11 / 1]$} & {$[1 / 11]$} & $f$ \\
\hline$\eta_{\|}$ & 2.27 & 1.67 & 2.00 & 1.681 & 1.725 & 1.541 & 1.594 & 1.429 & 1.428 & 1.429 \\
$\eta_{\perp}$ & 2.74 & 1.91 & 1.00 & 0.841 & 0.863 & 0.783 & 0.805 & 0.749 & 0.749 & 0.749 \\
$\Delta_{1}$ & 2.32 & 3.69 & 0.25 & 0.409 & 0.440 & 0.478 & 0.504 & 0.530 & 0.530 & 0.530 \\
$\beta_{1}$ & -4.39 & -2.58 & 0.75 & 0.909 & 0.940 & 0.873 & 0.858 & 0.880 & 0.880 & 0.880 \\
$\gamma_{11}$ & 0.00 & 0.00 & -0.50 & -0.50 & -0.50 & -0.433 & -0.428 & - & - & -0.400 \\
$\gamma_{1}$ & 3.38 & 17.70 & 0.50 & 0.739 & 0.814 & 0.810 & 0.838 & 0.839 & 0.837 & 0.838 \\
$\delta_{1}$ & 1.99 & 2.53 & 1.67 & 1.844 & 1.865 & 1.933 & 1.957 & 2.023 & 2.023 & 2.023 \\
$\delta_{11}$ & 1.83 & 2.47 & 0.33 & 0.475 & 0.498 & 0.552 & 0.582 & 0.647 & 0.647 & 0.647
\end{tabular}

TABLE II: Surface critical exponents of the ordinary transition for $d=3$ up to two-loop order at the cubic fixed point (of order $p=6) u^{*}=1.321(18), v^{*}=0.096(20)$, at $n=3$.

\begin{tabular}{rrrrrrrrrrr}
\hline $\exp$ & $\frac{O_{1}}{O_{2}}$ & $\frac{O_{1 i}}{O_{2 i}}$ & {$[0 / 0]$} & {$[1 / 0]$} & {$[0 / 1]$} & {$[2 / 0]$} & {$[0 / 2]$} & {$[11 / 1]$} & {$[1 / 11]$} & $f$ \\
\hline$\eta_{\|}$ & 2.29 & 1.68 & 2.00 & 1.684 & 1.727 & 1.545 & 1.597 & 1.436 & 1.435 & 1.436 \\
$\eta_{\perp}$ & 2.76 & 1.92 & 1.00 & 0.842 & 0.863 & 0.785 & 0.806 & 0.752 & 0.752 & 0.752 \\
$\Delta_{1}$ & 2.34 & 3.72 & 0.25 & 0.408 & 0.438 & 0.476 & 0.501 & 0.526 & 0.526 & 0.526 \\
$\beta_{1}$ & -4.42 & -2.60 & 0.75 & 0.908 & 0.938 & 0.872 & 0.858 & 0.879 & 0.879 & 0.879 \\
$\gamma_{11}$ & 0.00 & 0.00 & -0.50 & -0.50 & -0.50 & -0.433 & -0.428 & - & - & -0.424 \\
$\gamma_{1}$ & 3.41 & 17.87 & 0.50 & 0.737 & 0.811 & 0.807 & 0.834 & 0.835 & 0.836 & 0.836 \\
$\delta_{1}$ & 2.01 & 2.55 & 1.67 & 1.842 & 1.863 & 1.930 & 1.953 & 2.018 & 2.017 & 2.018 \\
$\delta_{11}$ & 1.84 & 2.48 & 0.33 & 0.474 & 0.497 & 0.550 & 0.579 & 0.642 & 0.642 & 0.642
\end{tabular}

TABLE III: Surface critical exponents of the ordinary transition for $d=3$ up to two-loop order at the cubic fixed point (of order $p=2) u^{*}=1.064, v^{*}=0.520$, at $n=4$.

\begin{tabular}{rrrrrrrrrrr}
\hline $\exp$ & $\frac{O_{1}}{O_{2}}$ & $\frac{O_{1 i}}{O_{2 i}}$ & {$[0 / 0]$} & {$[1 / 0]$} & {$[0 / 1]$} & {$[2 / 0]$} & {$[0 / 2]$} & {$[11 / 1]$} & {$[1 / 11]$} & $f$ \\
\hline$\eta_{\|}$ & 2.12 & 1.54 & 2.00 & 1.647 & 1.700 & 1.481 & 1.550 & 1.319 & 1.314 & 1.317 \\
$\eta_{\perp}$ & 2.51 & 1.74 & 1.00 & 0.824 & 0.850 & 0.753 & 0.783 & 0.705 & 0.705 & 0.705 \\
$\Delta_{1}$ & 2.07 & 3.27 & 0.25 & 0.426 & 0.464 & 0.511 & 0.549 & 0.588 & 0.589 & 0.589 \\
$\beta_{1}$ & -4.95 & -2.64 & 0.75 & 0.926 & 0.964 & 0.891 & 0.873 & 0.898 & 0.900 & 0.899 \\
$\gamma_{11}$ & 0.0 & 0.0 & -0.50 & -0.50 & -0.50 & -0.409 & -0.400 & - & -0.311 \\
$\gamma_{1}$ & 2.91 & 12.61 & 0.50 & 0.765 & 0.860 & 0.856 & 0.900 & 0.899 & 0.902 & 0.901 \\
$\delta_{1}$ & 1.83 & 2.34 & 1.67 & 1.863 & 1.889 & 1.969 & 2.003 & 2.105 & 2.103 & 2.104 \\
$\delta_{11}$ & 1.70 & 2.31 & 0.33 & 0.490 & 0.519 & 0.583 & 0.623 & 0.727 & 0.724 & 0.726
\end{tabular}

* Electronic address: pylyp@ph.icmp.lviv.ua

1 A. Aharony, Phys. Rev. B 8, 4270 (1973).

2 I.J. Ketley and D.J. Wallace, J.Phys.A 6,1667 (1973).

3 D.J. Wallace, J. Phys. C 6, 1390 (1973).

${ }^{4}$ E. Brézin, J. C. Le Guillou, J.Zinn-Justin, Phys.Rev.B 10, 892 (1974).

${ }^{5}$ G. Grinstein and A. Luther, Phys. Rev. B 13, 1329 (1976). 
TABLE IV: Surface critical exponents of the ordinary transition for $d=3$ up to two-loop order at the cubic fixed point (of order $p=2) u^{*}=0.525, v^{*}=1.146$, at $n=8$.

\begin{tabular}{rrrrrrrrrrr}
\hline $\exp$ & $\frac{O_{1}}{O_{2}}$ & $\frac{O_{1 i}}{O_{2 i}}$ & {$[0 / 0]$} & {$[1 / 0]$} & {$[0 / 1]$} & {$[2 / 0]$} & {$[0 / 2]$} & {$[11 / 1]$} & {$[1 / 11]$} & $f$ \\
\hline$\eta_{\|}$ & 2.13 & 1.55 & 2.00 & 1.645 & 1.699 & 1.479 & 1.548 & 1.307 & 1.297 & 1.302 \\
$\eta_{\perp}$ & 2.51 & 1.74 & 1.00 & 0.823 & 0.849 & 0.752 & 0.781 & 0.702 & 0.701 & 0.702 \\
$\Delta_{1}$ & 2.05 & 3.22 & 0.25 & 0.427 & 0.466 & 0.514 & 0.553 & 0.592 & 0.593 & 0.593 \\
$\beta_{1}$ & -5.45 & -2.77 & 0.75 & 0.927 & 0.966 & 0.895 & 0.878 & 0.903 & 0.905 & 0.904 \\
$\gamma_{11}$ & 0.0 & 0.0 & -0.50 & -0.50 & -0.50 & -0.393 & -0.380 & - & - & -0.315 \\
$\gamma_{1}$ & 2.84 & 11.57 & 0.50 & 0.766 & 0.863 & 0.860 & 0.907 & 0.902 & 0.909 & 0.906 \\
$\delta_{1}$ & 1.84 & 2.34 & 1.67 & 1.864 & 1.890 & 1.971 & 2.005 & 2.113 & 2.139 & 2.126 \\
$\delta_{11}$ & 1.70 & 2.33 & 0.33 & 0.491 & 0.521 & 0.584 & 0.624 & 0.739 & 0.732 & 0.736
\end{tabular}

TABLE V: Surface critical exponents of the special transition for $d=3$ up to two-loop order at the cubic fixed point (of order $p=2) u^{*}=0.201, v^{*}=1.508$, for $n \rightarrow \infty$.

\begin{tabular}{rrrrrrrrrrr}
\hline $\exp$ & $\frac{O_{1}}{O_{2}}$ & $\frac{O_{1 i}}{O_{2 i}}$ & {$[0 / 0]$} & {$[1 / 0]$} & {$[0 / 1]$} & {$[2 / 0]$} & {$[0 / 2]$} & {$[11 / 1]$} & {$[1 / 11]$} & $f$ \\
\hline$\eta_{\|}$ & 2.14 & 1.56 & 2.00 & 1.648 & 1.701 & 1.484 & 1.552 & 1.312 & 1.300 & 1.306 \\
$\eta_{\perp}$ & 2.53 & 1.75 & 1.00 & 0.824 & 0.850 & 0.755 & 0.783 & 0.706 & 0.704 & 0.705 \\
$\Delta_{1}$ & 2.07 & 3.26 & 0.25 & 0.426 & 0.463 & 0.511 & 0.549 & 0.585 & 0.587 & 0.586 \\
$\beta_{1}$ & -5.34 & -2.75 & 0.75 & 0.926 & 0.963 & 0.893 & 0.876 & 0.901 & 0.904 & 0.903 \\
$\gamma_{11}$ & 0.0 & 0.0 & -0.50 & -0.50 & -0.50 & -0.387 & -0.373 & - & - & -0.323 \\
$\gamma_{1}$ & 2.88 & 11.93 & 0.50 & 0.764 & 0.858 & 0.856 & 0.901 & 0.895 & 0.902 & 0.899 \\
$\delta_{1}$ & 1.85 & 2.36 & 1.67 & 1.862 & 1.888 & 1.968 & 2.001 & 2.108 & 2.105 & 2.107 \\
$\delta_{11}$ & 1.71 & 2.34 & 0.33 & 0.490 & 0.519 & 0.581 & 0.621 & 0.736 & 0.728 & 0.732
\end{tabular}

6 A.B.Harris, Z. Phys. B: Condens.Matter 49, 347 (1983).

7 Y.Kim, J. Phys. C 16, 1345 (1983).

8 A. Aharony, Phys. Rev. Lett. 31, 1494 (1973).

9 J.Sznajd, J. Magn. Matter 42,269 (1984); Z.Domański and J.Sznajd, Phys. Status Solidi B 129,135 (1985); J.Sznajd and M.Dudziński, Phys. Rev. B 59, 4176 (1999).

10 K. Binder, in Phase Transitions and Critical Phenomena, edited by C. Domb and J. L. Lebowitz (Academic Press, London, 1983), Vol. 8, pp. 1-144.

11 H. W. Diehl, in Phase Transitions and Critical Phenomena, edited by C. Domb and J. L. Lebowitz (Academic Press, London, 1986), Vol. 10, pp. 75-267.

12 H.W.Diehl, Int.J.Mod.Phys.B 11, 3503 (1997); preprint cond-mat/9610143.

13 K. Binder, P.C. Hohenberg, Phys.Rev.B 6, 3461 (1972); Phys.Rev.B 9, 2194 (1974).

${ }^{14}$ H.W.Diehl and S.Dietrich, Z. Phys. B: Condens. Matter 42, 65 (1981).

15 H.W.Diehl,E.Eisenriegler, Phys.Rev.Lett. 48, 1767 (1982).

16 H.W.Diehl,E.Eisenriegler, Phys.Rev.B 30, 300 (1984).

17 H.W.Diehl and S.Dietrich, Phys. Rev. B 24, 2878 (1981); Z. Phys. B 50, 117 (1983).

18 H.W.Diehl, S.Dietrich, and E.Eisenriegler, Phys. Rev. B 27, 2937 (1983).

19 H.W.Diehl, A.Ciach, Phys.Rev.B 44, 6642 (1991).

${ }^{20}$ H. W. Diehl and M. Shpot, Nucl. Phys. B 528, 595 (1998).

${ }^{21}$ H. W. Diehl and A. Nüsser, Z. Phys. B 79, 69 (1990); Z. Phys. B 79, 79 (1990).

${ }^{22}$ M. Pleimling and W. Selke, Eur. Phys. J. B 1, 385 (1998).

23 H. W. Diehl, Eur.Phys.J. B 1, 401 (1998).

24 Z.E. Usatenko, M.A. Shpot, Chin-Kun Hu, Phys. Rev. E 63, 056102 (2001).

25 Z.Usatenko and Chin-Kun Hu, Phys. Rev. E 65, 066103 (2002).

${ }^{26}$ F.Iglói, P.Lajkó, W. Selke and F. Szalma, J. Phys. A 31, 2801 (1998).

27 E.Brezin and S.Leibler, Phys.Rev.B 27, 594 (1983).

28 U.Ritschel and P.Czerner, Phys.Rev.Lett. 77, 3645 (1996).

29 A.Ciach and U.Ritschel, Nucl.Phys.B 489, 653 (1997).

30 A.Maciołek, A.Ciach, and A.Drzewinski, Phys.Rev.E 60, 2887 (1999). 
31 A.Drewitz, R.Leidl, T.W.Burkhardt, H.W.Diehl, Phys.Rev.Lett. 78, 1090 (1997).

32 Z.Usatenko and Chin-Kun Hu, Phys.Rev.E 68, (2003).

33 I.J. Ketley and D.J. Wallace, J.Phys. A 6, 1667 (1973).

34 A. Aharony and A.D. Bruce, Phys.Rev.Lett. 33, 427 (1974).

35 I.O. Mayer and A.I. Sokolov, Izv. Akad. Nauk SSSR, Ser. Fiz. 51, 2103 (1987).

36 N.A.Shpot, Phys. Lett. A, 142, 474 (1989).

37 M.C. Yalabik and A. Houghton, Phys. Lett. 61A, 1 (1977).

38 K.E. Newman and E.K. Riedel, Phys. Rev. B 25, 264 (1982).

39 I.O.Mayer, A.I.Sokolov, and B.N.Shalaev, Ferroelectrics 95, 93 (1989); I.O.Mayer, J. Phys. A 22, 2815 (1989).

${ }^{40}$ K. B. Varnashev, J.Phys. A 33,3121 (2000); Phys. Rev. B 61, 14660 (2000).

41 H. Kleinert, V. Schulte-Frohlinde, Phys.Lett. B 342, 284 (1995).

42 B. Shalaev, S.A.Antonenko, and A.I.Sokolov, Phys. Lett. A 230, 105 (1997).

43 J.M. Carmona, A. Pelissetto, E. Vicari, Phys. Rev. B 61, 15136 (2000).

44 G.A.Baker, Jr., Essentials of Padé Approximants (Academic, New York, 1975).

45 G.A.Baker, Jr., B.G. Nickel, M.S.Green, and D.I.Meiron, Phys. Rev. Lett. 36, 1351 (1976).

46 H.W.Diehl and S.Dietrich, Phys. Lett. bf 80A, 408 (1980).

47 E. Brézin, J. C. Le Guillou, J.Zinn-Justin, Phase Transition and Critical Phenomena, ed.by C.Domb and M.S.Green, V.6 (Academic Press, New York, 1976).

48 G. Parisi, J. Stat. Phys. 23, 49 (1980).

49 D.V.Pakhnin, A.I.Sokolov, Phys.Rev.B 61, 15130 (2000).

50 A. Pelissetto and E. Vicari, Phys. Rev. B 62, 6393 (2000).

51 M. Shpot, Cond. Mat. Phys. N 10, 143 (1997).

52 D.J. Amit, Field Theory, The Renormalization group and Critical Phenomena (World Scientific, Singapore, 1984).

53 J.Zinn-Justin, Euclidean Field Theory and Critical Phenomena (Oxford Univ. Press, New York, 1989).

${ }^{54}$ G.Parisi, Statistical Field Theory (Addison-Wesley, Redwood City, 1988).

55 C.Itzykson and J.-M. Drouffe, Statistical Field Theory, Vol.I (Cambridge Univ. Press, Cambridge, 1989).

56 C.Bagnuls and C.Bervillier, Phys.Rev.B 24, 1226 (1981).

57 H.W.Diehl and M.Shpot, Phys. Rev. Lett. 73, 3431 (1994).

58 This is an intuitive picture conveyed from the theory of bulk regular systems. Much less is known about the large-order behavior of perturbative expansions pertaining to infinite random systems (see Refs. ${ }^{59,60,61}$ ), especially at large space dimensionalities. At the present time, there are no explicit results on large orders for the surface quantities, even in the absence of disorder.

59 A. J. Bray et al., Phys. Rev. B 36, 2212 (1987).

60 A. J. McKane, Phys.Rev.B 49, 12003 (1994).

61 G. Álvarez, V. Martin-Mayor, and J. J. Ruiz-Lorenzo, J. Phys. A 33, 841 (2000).

62 G.A.Baker, Jr., B.G. Nickel and D.I.Meiron, Phys. Rev. B 17, 1365 (1978).

${ }^{63}$ We applied the formulas of $\beta$ functions, presented in $^{64}$ for the case of cubic anisotropic model with $m=1$ and $n=3$.

64 N.A.Shpot, Phys. Lett. A 133, 125 (1988).

${ }^{65}$ The values of $\nu$ and $\eta$ at $n=4,8$ and $n \rightarrow \infty$ are calculated from formulas, presented in ${ }^{64}$ for the case of cubic anisotropic model.

${ }^{66}$ In order to evaluate the difference between surface critical exponents of semi-infinite model with cubic anisotropy and surface critical exponents of standard semi-infinite $n$-component model ${ }^{14,17,20,57}$ we performed additional calculation for surface critical exponents on the basis of formulas from ${ }^{20,57}$ in the case of $3 \mathrm{D}$ semi-infinite model with $n=3$.

67 G. Jug, Phys. Rev. B 27, 609 (1983).

68 The investigation of the surface critical behavior of random semi-infinite systems at the special and ordinary surface transition was performed early in serial works ${ }^{24,25}$. The obtained results indicate that the surface critical exponents of random semiinfinite systems belong to the universality class of random fixed point. 